

\title{
Statistical model-based damage detection and localization: subspace-based residuals and damage-to-noise sensitivity ratios
}

\author{
Michèle Basseville, Laurent Mevel, Maurice Goursat
}

\section{To cite this version:}

Michèle Basseville, Laurent Mevel, Maurice Goursat. Statistical model-based damage detection and localization: subspace-based residuals and damage-to-noise sensitivity ratios. [Research Report] RR4645, INRIA. 2002. inria-00071940

\section{HAL Id: inria-00071940 \\ https://hal.inria.fr/inria-00071940}

Submitted on 23 May 2006

HAL is a multi-disciplinary open access archive for the deposit and dissemination of scientific research documents, whether they are published or not. The documents may come from teaching and research institutions in France or abroad, or from public or private research centers.
L'archive ouverte pluridisciplinaire HAL, est destinée au dépôt et à la diffusion de documents scientifiques de niveau recherche, publiés ou non, émanant des établissements d'enseignement et de recherche français ou étrangers, des laboratoires publics ou privés. 


\title{
Statistical model-based damage detection and localization: subspace-based residuals and damage-to-noise sensitivity ratios
}

\author{
Michèle Basseville, Laurent Mevel, Maurice Goursat
}

\section{$N^{\circ} 4645$}

Novembre 2002

THÈME 4 



\title{
RINRIA
}

\section{Statistical model-based damage detection and localization: subspace-based residuals and damage-to-noise sensitivity ratios}

\author{
Michèle Basseville* ${ }^{*}$ Laurent Mevel ${ }^{\dagger}$, Maurice Goursat ${ }^{\ddagger}$ \\ Thème 4 - Simulation et optimisation \\ de systèmes complexes \\ Projet Sigma2
}

Rapport de recherche $\mathrm{n}^{\circ} 4645$ - Novembre 2002 - 42 pages

\begin{abstract}
The vibration-based structural health monitoring problem is addressed as the double task of detecting damages modeled as changes in the eigenstructure of a linear dynamic system, and localizing the detected damages within (a FEM of) the monitored structure. The proposed damage detection algorithm is based on a residual generated from a stochastic subspace-based covariance-driven identification method and on the statistical local approach to the design of detection algorithms. This algorithm basically computes a global test, which performs a sensitivity analysis of the residuals to the damages, relative to uncertainties and noises. How this residual relates to some residuals for damage localization and model updating is discussed. Damage localization is stated as a detection problem. This problem is addressed by plugging aggregated sensitivities of the modes and mode-shapes w.r.t. FEM structural parameters in the above setting. This results in directional tests, which perform the same type of damage-to-noise sensitivity analysis of the residual as for damage detection. How the sensitivity aggregation mechanism relates to substructuring is outlined. Numerical results obtained on one example are reported.
\end{abstract}

Key-words: Damage detection, damage localization, vibration analysis, subspace algorithms, statistical local approach, sensitivity.

(Résumé : tsvp)

\footnotetext{
* IRISA/CNRS - basseville@irisa.fr.

$\dagger$ IRISA/INRIA - Laurent.Mevel@irisa.fr.

$\ddagger$ INRIA - Maurice.Goursat@inria.fr.
} 


\section{Approche statistique paramétrique pour la détection et la localisation d'endommagements: résidus de type sous-espace et sensibilités relatives.}

Résumé : Le problème de la surveillance de l'intégrité de structures en vibrations est décomposé en deux tâches de détection et de localisation d'endommagements. La première est traitée comme un problème de détection de changements dans la structure propre d'un système dynamique, et la deuxième repose sur l'utilisation d'un modèle éléments finis.

L'algorithme de détection d'endommagements proposé comporte le calcul d'un résidu généré à partir de la méthode d'identification par sous-espace à base de covariances, et évalué au moyen de l'approche statistique locale de la conception d'algorithmes de détection. Cet algorithme calcule essentiellement un test global, qui effectue une analyse des sensibilités du résidu aux endommagements, relativement aux incertitudes et aux bruits présents sur le système. Le lien entre ce résidu et d'autres résidus introduits dans la littérature pour la localisation d'endommagements et le recalage de modèles est discuté.

Le problème de la localisation d'endommagements est posé comme un problème de détection, et non comme un problème (inverse) d'estimation. Il est abordé à l'aide des sensibilités des modes et déformées modales vis-à-vis des paramètres structuraux du modèle éléments finis. Parce que tous les endommagements élémentaires envisageables ne peuvent pas être discriminés lors d'une surveillance avec un petit nombre de capteurs et un petit nombre de modes propres de référence, ces sensibilités sont aggrégées à l'aide d'une métrique cohérente avec la métrique du test global. Les sensibilités ainsi aggrégées sont ensuite injectées dans le formalisme de test précédent.

Mots-clé : Détection d'endommagements, localisation d'endommagements, analyse des vibrations, algorithmes de type sous-espace, approche asymptotique locale, sensibilités. 


\section{Introduction}

Detecting and localizing damages for monitoring the integrity of structural and mechanical systems is a topic of growing interest, due to the aging of many engineering constructions and machines and to increased safety norms. Automatic global vibration-based monitoring techniques turn out to be useful alternatives to visual inspections or local non destructive (e.g. ultrasonic) evaluations performed manually.

Health monitoring techniques based on processing vibration measurements basically handle two types of characteristics: the structural parameters (mass, stiffness, flexibility, damping) and the modal parameters (modal frequencies, and associated damping values and mode-shapes); see $[1,2,3]$ and references therein. A central question for monitoring is to compute changes in those characteristics and to assess their significance. For the frequencies, crucial issues are then: how to compute the changes, to assess that the changes are significant, to handle correlations among individual changes. A related issue is how to compare the changes in the frequencies obtained from experimental data with the sensitivity of modal parameters obtained from an analytical model. Furthermore, it has been widely acknowledged that, whereas changes in frequencies bear useful information for damage detection, information on changes in (the curvature of) mode-shapes is mandatory for performing damage localization. Then, similar issues arise for the computation and the significance of the changes. In particular, assessing the significance of (usually small) changes in the mode-shapes, and handling the (usually high) correlations among individual mode-shape changes are still considered as opened questions $[1,2,3,4]$.

Controlling the computational complexity of the processing of the collected data is another standard monitoring requirement, which includes a limited use of an analytical model of the structure. Moreover, the reduction from the analytical model to the experimental model (truncated modal space) is known to play a key role in the success of model-based damage detection and localization $[1,5]$.

The purpose of the present paper is to describe the foundations and analyze the properties of a damage detection and localization method. This method is based on an approach which aims at addressing the issues and overcoming (some of) the limitations above. It is assumed that a signature of the structure in its nominal (safe) state is available, typically a moderate number of modes and a moderate number of components of associated mode-shapes. This signature is usually identified using reference data, possibly recorded under an unknown non-stationary excitation. The proposed algorithm processes new data by first generating a residual, and computing its sensitivity w.r.t. damages. The residual is shown to be asymptotically Gaussian

$\mathrm{RR} \mathrm{n}^{\circ} 4645$ 
under both no damage and small damage assumptions. This results in a global test, which performs a sensitivity analysis of the residual to the damages, relative to uncertainties in the modal estimates and noises on the available data. Modal diagnosis is stated as a detection problem: decide which components of the modal parameter vector $\theta$ have changed. This problem is solved by designing similar $\chi^{2}$-tests focussed onto the modal subspaces of interest. Damage localization is stated as a detection problem, and not an (usually ill-posed) inverse estimation problem. This problem is addressed by plugging aggregated sensitivities of the modes and mode-shapes w.r.t. FEM structural parameters in the above setting. This results in directional tests, which perform the same type of damage-to-noise sensitivity analysis of the residual as for damage detection. The computation, the analytical-to-experimental matching and the aggregation of the sensitivities are performed off-line at a design stage, whereas the directional tests may be computed on-board.

The paper is organized as follows. In section 2, we introduce the modeling and discuss some key parameterizations and sensor types issues. Section 3 is devoted to the proposed damage detection method, based on a general statistical local approach to the detection of small deviations in the parameters of dynamic systems, and on a stochastic subspace-based covariance-driven modal identification method. The off-line computation and the on-board analysis stage are distinguished, and the effect of a truncated modal space is briefly discussed. In section 4, we describe the modal diagnosis method, which handles residual sensitivities to damages and residuals uncertainties. Damage localization is addressed in section 5, where the off-line computation and the on-board analysis stage are distinguished again. How the proposed method relates to other works is addressed in section 6 , where residual design and structural aggregation are discussed. Numerical results obtained on two application examples are reported in section 7 . Some conclusions are drawn in section 8 .

\section{Modeling and parameterizations}

We first recall the main equations and parameters of the models we use. Identifiable and non identifiable models are distinguished, and a useful invariance property of the modal parameters is outlined. Then the effect of changing the sensor types is discussed. Finally, the damage detection and localization problems investigated throughout are stated. 


\subsection{Dynamical model and structural parameters}

We assume that the behavior of the mechanical system can be described by a stationary linear dynamical system, and that, in the frequency range of interest, the input forces can be modeled as a non-stationary white noise. This results in:

$$
M \ddot{\mathcal{Z}}(t)+C \dot{\mathcal{Z}}(t)+K \mathcal{Z}(t)=\nu(t), \quad Y(t)=L \mathcal{Z}(t)
$$

where $t$ denotes continuous time, $M, C, K$ are the mass, damping and stiffness matrices respectively, (high dimensional) vector $\mathcal{Z}$ collects the displacements of the degrees of freedom of the structure; the external (non measured) force $\nu$ is modeled as a non-stationary white noise with time-varying covariance matrix $Q_{\nu}(t)$, measurements are collected in the (often, low dimensional) vector $Y$, and matrix $L$ indicates which components of the state vector are actually measured (where the sensors are located).

The modes or eigenfrequencies denoted generically by $\mu$, the eigenvectors $\phi_{\mu}$, and the mode-shapes denoted generically by $\psi_{\mu}$, are solutions of:

$$
\operatorname{det}\left(\mu^{2} M+\mu C+K\right)=0, \quad\left(\mu^{2} M+\mu C+K\right) \phi_{\mu}=0, \quad \psi_{\mu}=L \phi_{\mu}
$$

The frequency and damping coefficient are recovered from a continuous eigenvalue $\mu$ through:

$$
\text { Frequency } f=\frac{b}{2 \pi} \text {, Damping } d=-\frac{a}{\sqrt{a^{2}+b^{2}}} \text {, where: } a=\Re(\mu), \quad b=\Im(\mu)
$$

A local damage in the structure reduces the stiffness and increases the damping. Thus many damage detection techniques have been proposed which monitor the stiffness matrix $K$. However, monitoring its inverse $K^{-1}$, namely the flexibility matrix, has proven more tractable and computationally feasible $[1,4,5]$. In some cases, other structural parameterizations such as volumic mass and Young elasticity modulus may be preferable $[6,1]$. Several methods in the literature are based on a transmissibility matrix [3,7], which involves the processing of input-output data. However, in the case of non measured input excitation, processing output-only data is mandatory $[8,9]$. On the other hand, a reduced stiffness and an increased damping result in decreased natural frequencies and modified mode-shapes geometry. Monitoring the modal parameters is thus relevant, at least for damage detection. Damage localization, however, requires (at least partial) knowledge of structural parameters and geometry.

The proposed damage detection method handles the modal parameters, which enjoy a useful invariance property, as explained next. Moreover, the proposed method 
does not make use of any modal parameters extracted from an analytical model, but uses identified modal parameters instead. The proposed damage localization method handles both modal and structural parameters, using an original structural aggregation mechanism.

\subsection{State-space model and canonical parameterization}

Sampling model (1) at rate $1 / \tau$ yields the discrete time model in state space form $[10,11]$ :

$$
\left\{\begin{aligned}
X_{k+1} & =F X_{k}+V_{k+1} \\
Y_{k} & =H X_{k}
\end{aligned}\right.
$$

where the state and the output are:

$$
X_{k}=\left(\begin{array}{c}
\mathcal{Z}(k \tau) \\
\dot{\mathcal{Z}}(k \tau)
\end{array}\right), Y_{k}=Y(k \tau),
$$

the state transition and observation matrices are:

$$
F=e^{\mathcal{L} \tau}, \mathcal{L}=\left(\begin{array}{cc}
0 & I \\
-M^{-1} K & -M^{-1} C
\end{array}\right)
$$

and

$$
H=\left(\begin{array}{ll}
L & 0
\end{array}\right)
$$

In (4), the unmeasured state noise $V_{k+1}$ is assumed to be Gaussian, zero-mean, white, with covariance matrix:

$$
Q_{k+1} \stackrel{\text { def }}{=} \mathbf{E}\left(V_{k+1} V_{k+1}^{T}\right)=\int_{k \tau}^{(k+1) \tau} e^{\mathcal{L} s} \widetilde{Q}(s) e^{\mathcal{L}^{T} s} d s
$$

where $\mathbf{E}($.$) denotes the expectation operator and$

$$
\widetilde{Q}(s)=\left(\begin{array}{cc}
0 & 0 \\
0 & M^{-1} Q_{\nu}(s) M^{-T}
\end{array}\right)
$$

The whiteness assumption on the state noise and the absence of measurement noise in (4) are discussed in [12]. It is stressed that sinusoidal or colored noise excitation can be encompassed as well. State $X$ and observed output $Y$ have dimensions $2 m$ and $r$ respectively, with $r$ (often much) smaller than $2 m$ in practice. 
Let $\left(\lambda, \phi_{\lambda}\right)$ be the eigenstructure of the state transition matrix $F$, namely:

$$
\operatorname{det}(F-\lambda I)=0, \quad(F-\lambda I) \phi_{\lambda}=0
$$

The modal parameters $\left(\mu, \psi_{\mu}\right)$ in (2) can be deduced from the $\left(\lambda, \phi_{\lambda}\right)$ 's in (7) using:

$$
e^{\tau \mu}=\lambda, \quad \psi_{\mu}=\varphi_{\lambda} \stackrel{\text { def }}{=} H \phi_{\lambda}
$$

The frequency and damping coefficient are recovered from a discrete eigenvalue $\lambda$ through:

$$
\text { Freq. }=\frac{a}{2 \pi \tau} \text {, Damping }=\frac{|b|}{\sqrt{a^{2}+b^{2}}} \text {, where: } a=\left|\arctan \frac{\Im(\lambda)}{\Re(\lambda)}\right|, b=\ln |\lambda|
$$

Eigenvectors are real if $C=\alpha M+\beta K$, the simplest form of proportional damping. Because of the structure of the state in (5), the $\lambda$ 's and $\varphi_{\lambda}$ 's are pairwise complex conjugate.

It turns out that the collection of modes $\left(\lambda, \varphi_{\lambda}\right)$, which form a very natural parameterization for structural analysis, also enjoy a nice invariance property. Actually, it can easily be shown to be invariant w.r.t. changes in the state basis of system (4). In other words, the $\left(\lambda, \varphi_{\lambda}\right)$ 's form a canonical parameterization of the eigenstructure (or equivalently the pole part) of that system. Let the $\left(\lambda, \varphi_{\lambda}\right)$ 's be stacked into a $(r+1) m$-dimensional vector $\theta$ :

$$
\theta \stackrel{\text { def }}{=}\left(\begin{array}{c}
\Lambda \\
\operatorname{vec} \Phi
\end{array}\right)
$$

where $\Lambda$ is the vector whose elements are the eigenvalues $\lambda, \Phi$ is the matrix whose columns are the mode-shapes $\varphi_{\lambda}$ 's, and vec is the column stacking operator. From now on, vector $\theta$ is considered as the system parameter.

We stress that we do not need to favor a particular normalization of the mode-shapes $\phi_{\mu}$ 's and thus of the $\varphi_{\lambda}$ 's, as opposite to the mass normalized modes: $\phi_{\mu}^{T} M \phi_{\mu}=I$ used in e.g. [5]. As explained below, the proposed damageto-noise sensitivity ratios are invariant w.r.t. such normalizations. However, some care should be taken for damage localization, while matching identified mode-shapes with analytical ones as discussed in 5.2.3.

\subsection{Different sensor types}

The measurement equation in (4) with $H$ as in (6) implicitly assumes that the available sensors measure the (relative) displacements of the degrees of freedom them- 
selves, namely are constraint gauges. If constraint gauges, velocity sensors and accelerometers are available, the measurement equation in (1) should write :

$$
Y(t)=\left(\begin{array}{c}
L \mathcal{Z}(t) \\
N \dot{\mathcal{Z}}(t) \\
P \ddot{\mathcal{Z}}(t)
\end{array}\right)
$$

with $L, N, P$ made of 0 's and 1's, and system (5) should be understood with :

$$
H=\left(\begin{array}{cc}
L & 0 \\
0 & N \\
-P M^{-1} K & -P M^{-1} C
\end{array}\right)
$$

Consequently, state space model (4) can always be enforced, whatever the sensors are. The nature of the sensors used only influences the observation matrix $H$.

\subsection{The damage detection and localization problems}

In this paper, damage detection is stated as the problem of detecting changes in the canonical parameter vector $\theta$ defined in (10). It is assumed that a reference value $\theta_{0}$ is available. Generally, such a reference parameter is identified using data recorded on the undamaged system. Of course, when the monitored system is subject to nonstationary input excitation, the reference value $\theta_{0}$ should be identified on long data samples containing as many of these nuisance changes as possible. However, it is important to note that, with the proposed method, the detection problem may be solved on the basis of data samples of much smaller size.

Given, on one hand, a reference value $\theta_{0}$ of the model parameter and, on the other hand, a new data sample, the detection problem is to decide whether the new data are still well described by this parameter value or not. The modal diagnosis problem is to decide which components of the modal parameter vector $\theta$ have changed. The damage localization problem is to decide which parts of the structure have been damaged, or equivalently to decide which elements of the structural parameter matrices have changed.

Because structural identification is a complex (and generally not fully automatic) process, and because it is intended to design a damage detection algorithm which can be run on-board, our approach does not perform a new parameter estimation using the new data sample. Instead, the damage detection and modal diagnosis problems are solved through the on-board computation and analysis of a residual. Similarly, 
because structural model updating is a computationally involved procedure, the damage localization problem is not addressed as an (usually ill-posed inverse) estimation problem, but as an evaluation of the correlations of this residual with specific structural parameter subspaces. This is explained in the next three sections.

\section{Damage detection}

The design of the proposed damage detection algorithm is based on a general statistical approach, which aims at transforming a large class of detection problems concerning a parameterized stochastic process into the universal problem of monitoring the mean of a Gaussian random vector [14]. This approach basically addresses the early warning of small deviations of the system parameter. The key idea is to define a convenient residual, tightly associated with a relevant parameter estimation method, and to compute the sensitivity of the residual w.r.t. damages (viewed as changes in the parameter vector) and the uncertainty in the residual due to process noise and estimation errors. Moreover, the residual can be shown to be asymptotically Gaussian. Hence the analysis of the residuals sensitivity to the damages relative to uncertainties and noises is easy: a sound decision rule can be designed for assessing whether the residual has significantly deviated from zero or not.

For structural vibration monitoring and damage detection, the main issue is thus the definition of a parameter estimating function associated with a modal identification algorithm. The use of an output-only and covariance-driven subspace-based stochastic identification has been advocated [9, 12]. The residual corresponding to this method is introduced in 3.1. The handling of the residual sensitivities and uncertainty is addressed in 3.2, and their off-line computation described in 3.3. The residual analysis and the resulting on-board damage detection algorithm are given in 3.4 .

\subsection{Residual associated with stochastic subspace identification}

The key steps of the subspace structural identification method are briefly recalled. A characterization of the modal parameter in (10) is exhibited, from which the proposed residual can be defined. Finally, the effect of a truncated modal space is discussed.

$\mathrm{RR} \mathrm{n}^{\circ} 4645$ 


\subsubsection{Output-only covariance-driven subspace identification}

Covariance-driven subspace identification of the eigenstructure $\left(\lambda, \varphi_{\lambda}\right)$ 's is based on the following steps. Let $R_{i} \stackrel{\text { def }}{=} \mathbf{E}\left(Y_{k} Y_{k-i}^{T}\right)$ and

$$
\mathcal{H}_{p+1, q} \stackrel{\text { def }}{=}\left(\begin{array}{cccc}
R_{0} & R_{1} & \vdots & R_{q-1} \\
R_{1} & R_{2} & \vdots & R_{q} \\
\vdots & \vdots & \vdots & \vdots \\
R_{p} & R_{p+1} & \vdots & R_{p+q-1}
\end{array}\right) \stackrel{\text { def }}{=} \operatorname{Hank}\left(R_{i}\right)
$$

be the output covariance and Hankel matrices, respectively. Introducing the crosscovariance between the state and the observed outputs: $G \stackrel{\text { def }}{=} \mathbf{E}\left(X_{k} Y_{k}^{T}\right)$, direct computations of the $R_{i}$ 's from the equations (4) lead to: $R_{i}=H F^{i} G$, and to the well known [15] factorization

$$
\mathcal{H}_{p+1, q}=\mathcal{O}_{p+1}(H, F) \mathcal{C}_{q}(F, G)
$$

where

$$
\mathcal{O}_{p+1}(H, F) \stackrel{\text { def }}{=}\left(\begin{array}{l}
H \\
H F \\
\vdots \\
H F^{p}
\end{array}\right) \quad \text { and } \quad \mathcal{C}_{q}(F, G) \stackrel{\text { def }}{=}\left(G F G \cdots F^{q-1} G\right)
$$

are the observability and controllability matrices, respectively. The observation matrix $H$ is then found in the first block-row of the observability matrix $\mathcal{O}$. The statetransition matrix $F$ is obtained from the shift invariance property of $\mathcal{O}$, namely:

$$
\mathcal{O}_{p}^{\uparrow}(H, F)=\mathcal{O}_{p}(H, F) F, \quad \text { where } \mathcal{O}_{p}^{\uparrow}(H, F) \stackrel{\text { def }}{=}\left(\begin{array}{l}
H F \\
H F^{2} \\
\vdots \\
H F^{p}
\end{array}\right)
$$

Assuming $\operatorname{rank}\left(\mathcal{O}_{p}\right)=\operatorname{dim} F$, and thus that the number of block-rows in $\mathcal{H}_{p+1, q}$ is large enough, is mandatory for recovering $F$. The eigenstructure $\left(\lambda, \phi_{\lambda}\right)$ then results from (7). 
The actual implementation of this subspace algorithm, known under the name of balanced realization (BR) $[16,17]$, processes the empirical covariance and Hankel matrices

$$
\widehat{R}_{i} \stackrel{\text { def }}{=} 1 / n \sum_{k=1}^{n} Y_{k} Y_{k-i}^{T}, \quad \widehat{\mathcal{H}}_{p+1, q} \stackrel{\text { def }}{=} \operatorname{Hank}\left(\widehat{R}_{i}\right)
$$

and exploits the well known subspace interpretation of the singular value decomposition (SVD) [18]: the SVD of $\widehat{\mathcal{H}}_{p+1, q}$ - possibly pre- and post-weighted [19] - and its truncation at the desired model order yield, in the left factor, an estimate $\widehat{\mathcal{O}}$ for the observability matrix $\mathcal{O}$ :

$$
\begin{aligned}
W_{1} \widehat{\mathcal{H}} W_{2}^{T} & =U D V^{T}=U\left(\begin{array}{cc}
D_{1} & 0 \\
0 & D_{0}
\end{array}\right) V^{T} \\
\widehat{\mathcal{O}} & =W_{1}^{-1} U D_{1}^{1 / 2}, \quad \widehat{\mathcal{C}}=D_{1}^{1 / 2} V^{T} W_{2}^{-T}
\end{aligned}
$$

where $W_{1}$ and $W_{2}$ are invertible weighting matrices (design parameters). From $\widehat{\mathcal{O}}$, estimates $(\widehat{H}, \widehat{F})$ and $\left(\widehat{\lambda}, \widehat{\phi}_{\lambda}\right)$ are recovered as sketched above. How to select the number of lags $(p+q)$ and thus the size of $\widehat{\mathcal{H}}_{p+1, q}$ is discussed in $[9,12]$.

The key feature in this algorithm is the factorization (12), where the left factor $\mathcal{O}$ only depends on the pair $(H, F)$, and thus on the modal parameters $\left(\lambda, \varphi_{\lambda}\right)$.

\subsubsection{Exploiting a canonical parameter characterization}

Factorization (12) is the key for a characterization of the canonical parameter vector $\theta$ in (10), and for deriving the parameter estimating function implicitly used in the above subspace identification algorithm.

Assume that the eigenvectors of matrix $F$ are chosen as a basis for the state space of model (4). In this basis, the observability matrix in (13) writes [20]:

$$
\mathcal{O}_{p+1}(\theta)=\left(\begin{array}{l}
\Phi \\
\Phi \Delta \\
\vdots \\
\Phi \Delta^{p}
\end{array}\right)
$$

where diagonal matrix $\Delta$ is defined as $\Delta=\operatorname{diag}(\Lambda)$, and $\Lambda$ and $\Phi$ are as in (10). Then the following property results from the factorization (12). Whether a nominal parameter $\theta_{0}$ is in agreement with a given output covariance sequence $\left(R_{j}\right)_{j}$ is 
characterized by [21, 22]:

$$
\mathcal{O}_{p+1}\left(\theta_{0}\right) \text { and } \mathcal{H}_{p+1, q} \text { have the same left kernel } \text { space }^{1} \text {. }
$$

This property can be checked as follows. From the nominal modal parameter vector $\theta_{0}$, compute $\mathcal{O}_{p+1}\left(\theta_{0}\right)$ using (15), and perform e.g. a SVD of $W_{1} \mathcal{O}_{p+1}\left(\theta_{0}\right)$ for defining its left kernel space, namely extracting an orthonormal matrix $S$ such that $S^{T} S=I_{s}$ and:

$$
S^{T} W_{1} \mathcal{O}_{p+1}\left(\theta_{0}\right)=0
$$

Matrix $S$ depends implicitly on parameter $\theta_{0}$. It is not unique - two such matrices are related through a post-multiplication with an orthonormal matrix $U$. Nevertheless, for reasons which are made clear below, $S$ can be treated as a function of $\theta_{0}$, denoted by $S\left(\theta_{0}\right)$. Then the characteristic property (16) writes:

$$
U^{T} S^{T}\left(\theta_{0}\right) W_{1} \mathcal{H}_{p+1, q} W_{2}^{T}=0
$$

where $W_{1}$ and $W_{2}$ are invertible weighting matrices as before.

Assume now that a reference parameter $\theta_{0}$ and a new data sample $Y_{1}, \ldots, Y_{n}$ are available. For checking whether the data are well described by $\theta_{0}$, the idea is to compute the empirical covariance sequence and fill in the empirical block-Hankel matrix $\widehat{\mathcal{H}}_{p+1, q}$ using (14), and to define the residual vector ${ }^{2}$ :

$$
\zeta_{n}\left(\theta_{0}\right) \stackrel{\text { def }}{=} \sqrt{n} \operatorname{vec}\left(U^{T} S^{T}\left(\theta_{0}\right) W_{1} \widehat{\mathcal{H}}_{p+1, q} W_{2}^{T}\right)
$$

Let $\theta$ be the actual value of the parameter for the system which generated the new data sample, and $\mathbf{E}_{\theta}$ be the expectation when the actual parameter is $\theta$. It results from (18) that:

$$
\mathbf{E}_{\theta}\left(\zeta_{n}\left(\theta_{0}\right)\right)=0 \quad \text { iff } \theta=\theta_{0}
$$

In other words, vector $\zeta_{n}\left(\theta_{0}\right)$ in (19) has zero mean in the absence of change in $\theta$, and nonzero mean in the presence of a change (damage). Consequently it plays the role of a residual. The question is then how to decide that the residual $\zeta_{n}$ is significantly different from zero. In particular, the sensitivity of the residual w.r.t. deviations in the modal parameter should be compared with the fluctuations of the residual around its zero mean.

\footnotetext{
${ }^{1}$ The left kernel space of matrix $M$ is the kernel space of matrix $M^{T}$.

${ }^{2}$ Technical arguments for the $\sqrt{n}$ factor can be found in [14].
} 


\subsubsection{On the effect of a truncated modal space}

Some issues in dealing with a truncated modal space are now addressed.

Monitoring with a few modes. Many practical situations correspond to the case where actual data are generated by a system of high order. Controlling the computational complexity of the processing of the collected data is a standard monitoring requirement, which includes the reduction from the analytical model to the experimental model. In the same spirit as a truncated modal space is handled when estimating a flexibility matrix from a few of the lower frequencies $[1,5]$, the actual computation of the residual (19) is achieved using a reference parameter vector $\theta_{0}$ containing only a moderate number of modes and a moderate number of components of associated mode-shapes.

Model reduction. The model reduction issue can be investigated further along the following lines. When the actual data are generated by a system of higher order than that of the nominal model $\theta_{0}$, or equivalently when the nominal model has reduced order, a new question arises : what does it mean, for a nominal model $\theta_{0}$, to match a given data sample when model reduction is enforced? Of course, system theoretic characterization (16), or (18), is no longer valid, and the same is true for the definition of the residual in (19). Other definitions are needed [22], as sketched now.

Since $\operatorname{rank} \mathcal{H}_{p+1, q}>\operatorname{rank} \mathcal{O}_{p+1}\left(\theta_{0}\right) \stackrel{\text { def }}{=} m$, condition (16) for perfect matching cannot be satisfied. What can be required, instead, is that the left kernel space of $\mathcal{O}_{p+1}\left(\theta_{0}\right)$ is orthogonal to the $m$-th order principal subspace ${ }^{3}$ of $\mathcal{H}_{p+1, q}$. Therefore, let

$$
W_{1} \mathcal{H}_{p+1, q} W_{2}^{T}=\left(\begin{array}{ll}
P_{m} & \overline{P_{m}}
\end{array}\right) D V^{T}
$$

where $P_{m}$ collects the first $m$ left singular vectors of $W_{1} \mathcal{H}_{p+1, q} W_{2}^{T}$. Then, (18) is replaced by

$$
U^{T} S^{T}\left(\theta_{0}\right) P_{m} D V^{T}=0,
$$

and the residual is computed as

$$
\zeta_{n}\left(\theta_{0}\right) \stackrel{\text { def }}{=} \sqrt{n} \operatorname{vec}\left(U^{T} S^{T}\left(\theta_{0}\right) \widehat{P}_{m} \widehat{D} \widehat{V}^{T}\right)
$$

with obvious notations, namely: $W_{1} \widehat{\mathcal{H}}_{p+1, q} W_{2}^{T}=\left(\widehat{P}_{m} \overline{\widehat{P}_{m}}\right) \widehat{D} \widehat{V}^{T}$.

\footnotetext{
${ }^{3}$ The $m$-th order principal subspace of a matrix is the subspace spanned by the left singular vectors associated with its $m$ largest singular values.
}

$\mathrm{RR} \mathrm{n}^{\circ} 4645$ 
It turns out that, because of our practical implementation of the residual (19), and especially how we select the integer index $p$ for $\mathcal{H}_{p+1, q}$, the use of the residual (22) above does not seem to lead to any significant performance improvement.

\subsection{Residual sensitivities and residual uncertainty}

A natural approach to analyze the effect of deviations is to compute sensitivities in terms of gradients. This has been advocated for vibration monitoring as well [2]. Since our approach deals with small deviations, computing gradients is especially relevant. Because the approach is statistical, it is natural to consider the mean value (expectation) of those gradients.

On the other hand, the sensitivity matrix of the residual w.r.t. damages should be examined in the light of the variance of (or the uncertainty in) the components of the residual vector. Within a statistical approach, it is also natural to take into account the possible correlations among those components.

This is done now. The off-line and on-board computation stages of the proposed method are distinguished, in 3.3 and 3.4 respectively.

\subsection{Off-line computations}

The sensitivity of the residual w.r.t. modal changes is computed, and shown to enjoy a useful invariance property w.r.t. the normalization of the mode-shapes. Then the computation of the residual covariance matrix is discussed.

\subsubsection{Computing the residual sensitivities to modal changes}

The mean sensitivity of residual $\zeta_{n}$ w.r.t. $\theta$ is defined as:

$$
\begin{aligned}
\mathcal{J}\left(\theta_{0}\right) & \stackrel{\text { def }}{=}-1 / \sqrt{n} \partial /\left.\partial \theta \mathbf{E}_{\theta_{0}} \zeta_{n}(\theta)\right|_{\theta=\theta_{0}} \\
= & +1 / \sqrt{n} \partial /\left.\partial \theta \mathbf{E}_{\theta} \zeta_{n}\left(\theta_{0}\right)\right|_{\theta=\theta_{0}}
\end{aligned}
$$

where the last equality holds true because of (20). From (24) and using (12), it can be shown that [22]:

$$
\mathcal{J}\left(\theta_{0}\right)=\left(W_{2} \otimes U^{T} S^{T}\left(\theta_{0}\right) W_{1}\right)\left(\mathcal{H}_{p+1, q}^{T} \mathcal{O}_{p+1}^{\dagger T}\left(\theta_{0}\right) \otimes I_{(p+1) r}\right) \mathcal{O}_{p+1}^{\prime}\left(\theta_{0}\right)
$$


where $\mathcal{O}_{p+1}^{\dagger}\left(\theta_{0}\right)$ is the pseudo-inverse of $\mathcal{O}_{p+1}\left(\theta_{0}\right)$, and where:

$$
\begin{aligned}
\mathcal{O}_{p+1}^{\prime}\left(\theta_{0}\right) & \stackrel{\text { def }}{=} \partial / \partial \theta \operatorname{vec} \mathcal{O}_{p+1}\left(\theta_{0}\right) \\
& =\left(\begin{array}{ccc|ccc}
\Lambda_{1}^{\prime(p)} \otimes \varphi_{1} & & 0 & \Lambda_{1}^{(p)} \otimes I_{r} & & 0 \\
& \ddots & & \ddots & \\
0 & & \Lambda_{m}^{\prime(p)} \otimes \varphi_{m} & 0 & & \Lambda_{m}^{(p)} \otimes I_{r}
\end{array}\right)
\end{aligned}
$$

with, for $1 \leq i \leq m$ :

$$
\begin{array}{cccccc}
\Lambda_{i}^{(p) T} & \stackrel{\text { def }}{=} & \left(\begin{array}{ccccc}
1 & \lambda_{i} & \lambda_{i}^{2} & \ldots & \lambda_{i}^{p}
\end{array}\right) \\
\Lambda_{i}^{\prime(p) T} & \stackrel{\text { def }}{=} & \left(\begin{array}{llllll}
0 & 1 & 2 & \lambda_{i} & \ldots & p \lambda_{i}^{p-1}
\end{array}\right)
\end{array}
$$

A consistent estimate $\widehat{\mathcal{J}}$, based on a data sample, results from substituting $\widehat{\mathcal{H}}$ for $\mathcal{H}$ in (25):

$$
\widehat{\mathcal{J}}\left(\theta_{0}\right)=\left(W_{2} \otimes U^{T} S^{T}\left(\theta_{0}\right) W_{1}\right)\left(\widehat{\mathcal{H}}_{p+1, q}^{T} \mathcal{O}_{p+1}^{\dagger T}\left(\theta_{0}\right) \otimes I_{(p+1) r}\right) \mathcal{O}_{p+1}^{\prime}\left(\theta_{0}\right)
$$

We stress that all the terms in (27) should be computed when the reference parameter $\theta_{0}$ is identified, and using the same reference data. Note also that matrix $\mathcal{O}_{p+1}^{\prime}\left(\theta_{0}\right)$ is full rank $(r+1) m$, as can be checked from (26).

Finally, because the modes and mode-shapes are pairwise complex conjugate, the actual implementation of the computation above should take advantage of the real and imaginary parts, as made explicit in the Appendix.

\subsubsection{The sensitivity is invariant w.r.t. mode-shapes normalization}

The sensitivity matrix $\mathcal{J}\left(\theta_{0}\right)$ and its estimate $\widehat{\mathcal{J}}\left(\theta_{0}\right)$ enjoy a practically useful invariance property: they do not depend on the particular normalization of the eigenvectors $\varphi_{\lambda}$ stacked in $\theta_{0}$ defined in (10). Actually, multiplying the $\varphi_{\lambda}$ 's by constant complex numbers amounts to post-multiply observability matrix $\mathcal{O}_{p+1}\left(\theta_{0}\right)$ in (15) by an invertible diagonal matrix $D$, to post-multiply matrix $\mathcal{O}_{p+1}^{\dagger T}\left(\theta_{0}\right)$ by $D^{-1}$, and to pre-multiply matrix $\mathcal{O}_{p+1}^{\prime}\left(\theta_{0}\right)$ by $\left(D \otimes I_{(p+1) r}\right)$. And all the terms in $D$ cancel out in (27).

It should be noted that this invariance property only holds true for damage detection. For damage localization, some care should be taken of mode-shapes normalization, as explained in section 5.2. 


\subsubsection{Computing the residual uncertainty}

The residual covariance matrix is:

$$
\Sigma\left(\theta_{0}\right) \stackrel{\text { def }}{=} \lim _{n \rightarrow \infty} \mathbf{E}_{\theta_{0}}\left(\zeta_{n} \zeta_{n}^{T}\right)
$$

where it is assumed that the limit exists. Matrix $\Sigma$ captures the uncertainty in $\zeta_{n}$ due to estimation errors. Actually, the covariance matrix of the error in estimating $\theta_{0}$ can be shown to be this $\Sigma\left(\theta_{0}\right)$ as well [23].

The estimation of covariance matrix $\Sigma$ is somewhat tricky [24]. The following estimate is used in the experiments below. Assuming the whole sample size is $N \approx$ $K \ell$, we partition the data sample in $K$ segments with size $\ell$, and compute

$$
\widehat{\Sigma}=\frac{1}{K \ell} \sum_{k=1}^{K} \zeta_{k} \zeta_{k}^{T}
$$

where $\zeta_{k}$ is the residual (19) computed on segment $k$, using the data sample $Y_{(k-1) \ell+1}, \ldots, Y_{k \ell}$.

It should be noted that this estimate contains the excitation, and thus is affected by changes in the excitation. Therefore, and this is confirmed in the asymptotic Gaussianity theorem below, it is preferable to estimate it after collecting a new data sample. However, for the sake of reducing the computational complexity, it is often estimated prior to testing, using data on the safe system. The drawback of the latter

approach over the former is that the $\chi^{2}$-test below may detect changes due to the excitation and not to the structural properties.

\subsection{On-board residual analysis}

Testing if $\theta=\theta_{0}$ holds true requires the knowledge of the probability distribution of $\zeta_{n}\left(\theta_{0}\right)$. Unfortunately, this distribution is generally unknown. One manner to circumvent this difficulty is to assume close hypotheses:

$$
\text { (Safe) } \mathbf{H}_{0}: \theta=\theta_{0} \quad \text { and } \quad \text { (Damaged) } \mathbf{H}_{1}: \theta=\theta_{0}+\delta \theta / \sqrt{n}
$$

where vector $\delta \theta$ is unknown, but fixed. Note that for large $n$, hypothesis $\mathbf{H}_{1}$ corresponds to small deviations in $\theta$. This is known under the name of statistical local approach, of which the main result is the following $[24,23,14]$. 


\subsubsection{The residual is Gaussian}

Provided that $\Sigma\left(\theta_{0}\right)$ is positive definite, the residual $\zeta_{n}$ in (19) is asymptotically Gaussian distributed with the same covariance matrix $\Sigma\left(\theta_{0}\right)$ under both $\mathbf{H}_{0}$ and $\mathbf{H}_{1}$; that is [22]:

$$
\zeta_{n}\left(\theta_{0}\right) \underset{n \rightarrow \infty}{\longrightarrow}\left\{\begin{array}{rll}
\mathcal{N}\left(0, \Sigma\left(\theta_{0}\right)\right) & \text { under } & \mathbf{H}_{0} \\
\mathcal{N}\left(\mathcal{J}\left(\theta_{0}\right) \delta \theta, \Sigma\left(\theta_{0}\right)\right) & \text { under } & \mathbf{H}_{1}
\end{array}\right.
$$

As seen in (30), a deviation $\delta \theta$ in the system parameter $\theta$ is reflected into a change in the mean value of residual $\zeta_{n}$, which switches from zero (in the undamaged case) to $\mathcal{J}\left(\theta_{0}\right) \delta \theta$, as expected, in case of small damage.

Note that matrices $\mathcal{J}\left(\theta_{0}\right)$ and $\Sigma\left(\theta_{0}\right)$ depend on neither the sample size $n$ nor the fault vector $\delta \theta$ in hypothesis $\mathbf{H}_{1}$. Thus we do not need to re-estimate them when testing the hypotheses after collecting a new data sample, they can be estimated prior to testing, using data on the safe system (exactly as the reference parameter $\theta_{0}$ ).

In case of non-stationary excitation, a similar result has proven, for scalar output signals, and with matrix $\Sigma$ estimated on newly collected data [25].

\subsubsection{On-board $\chi^{2}$-test for damage detection}

Let $\widehat{\mathcal{J}}$ and $\widehat{\Sigma}$ be consistent estimates of $\mathcal{J}\left(\theta_{0}\right)$ and $\Sigma\left(\theta_{0}\right)$, and assume additionally that $\mathcal{J}\left(\theta_{0}\right)$ is full column rank (f.c.r.). Then, thanks to $(30)$, deciding that residual $\zeta_{n}$ is significantly different from zero, stated as testing between the hypotheses $\mathbf{H}_{0}$ and $\mathbf{H}_{1}$ in (29), can be achieved with the aid of the following $\chi^{2}$-test statistics:

$$
\chi_{n}^{2} \stackrel{\text { def }}{=} \zeta_{n}^{T} \widehat{\Sigma}^{-1} \widehat{\mathcal{J}}\left(\widehat{\mathcal{J}}^{T} \widehat{\Sigma}^{-1} \widehat{\mathcal{J}}\right)^{-1} \widehat{\mathcal{J}}^{T} \widehat{\Sigma}^{-1} \zeta_{n}
$$

which should be compared to a threshold. In (31), the dependence on $\theta_{0}$ has been removed for simplicity. We stress that the only term which should be computed after data collection is the residual $\zeta_{n}$ defined in (19).

Test statistics $\chi_{n}^{2}$ is asymptotically distributed as a $\chi^{2}$-variable, with $\operatorname{rank}(\mathcal{J})$ degrees of freedom and with non-centrality parameter under $\mathbf{H}_{1}: \delta \theta^{T} \mathcal{J}^{T} \Sigma^{-1} \mathcal{J} \delta \theta$.

\subsubsection{The $\chi^{2}$-test is invariant w.r.t. design matrices}

It turns out that the test in (31) enjoys a nice invariance property w.r.t. the choice of the design matrices in the subspace-based approach. The three design matrices $U, W_{1}, W_{2}$ are made explicit in the notation $\zeta_{n ; U, W_{1}, W_{2}}\left(\theta_{0}\right)$ for the residual defined 
in (19). A straightforward calculation shows that:

$$
\zeta_{n ; U, W_{1}, W_{2}}=\left(W_{2} \otimes V_{1}\right) \zeta_{n ; I, I, I}
$$

where $\otimes$ is the Kronecker product, and $V_{1}$ is any invertible matrix such that:

$U^{T} S^{T}\left(\theta_{0}\right) W_{1}=V_{1} S^{T}\left(\theta_{0}\right)$; for example, $V_{1}=U^{T} S^{T}\left(\theta_{0}\right) W_{1} S\left(\theta_{0}\right)$ [26].

Now, if $\widetilde{\zeta} \stackrel{\text { def }}{=} A \zeta$, then: $\widetilde{\mathcal{J}}=A \mathcal{J}$ and $\widetilde{\Sigma}=A \Sigma A^{T}$, as can easily be checked, and using obvious notations. Therefore, if $\chi_{n ; U, W_{1}, W_{2}}^{2}$ denotes the $\chi^{2}$-test (31) associated with $\zeta_{n ; U, W_{1}, W_{2}}$, from (32) we get that:

$$
\chi_{n ; U, W_{1}, W_{2}}^{2}=\chi_{n ; I, I, I}^{2}
$$

since the invertible matrix $\left(W_{2} \otimes V_{1}\right)$ factors out in (31).

Thus, for damage detection, all the proposed subspace-based methods are equivalent, when using the true system order.

\section{Modal diagnosis}

Modal diagnosis consists in determining which eigenfrequencies and associated modeshapes have been affected by the damage. This problem may be addressed as an estimation problem, based on modal identification in the pre- and post-damage stages. Typically, the changes in the frequencies obtained from experimental data are then compared with the sensitivity of modal parameters obtained from an analytical model $[1,2]$.

In the proposed approach, modal diagnosis is stated as a detection problem instead. Moreover no analytical model is handled at this stage. The rationale is the same as for addressing the damage detection problem above: having a (usually identified) reference modal parameter $\theta_{0}$ in one hand and a new data sample in the other, decide which modes and mode-shapes have deviated from their reference values.

\subsection{Residual sensitivities and residual uncertainty}

As for the damage detection problem, in a statistical framework the key issue is again to assess the significance of the residual sensitivity to specified modal changes w.r.t. the residual uncertainty. In other words, directional tests, focussed in specific directions of the modal space, should be designed.

At this point, it should be noted that the sensitivity of the residual $\zeta_{n}$ w.r.t. a specified mode and associated mode-shape can be extracted as the corresponding columns of the Jacobian matrix $\mathcal{J}\left(\theta_{0}\right)$ of which an estimate is given in (27). 


\subsection{On-board $\chi^{2}$-test for modal diagnosis}

Let thus $\widehat{\mathcal{J}}_{i}$ be the estimated Jacobian matrix corresponding to mode and modeshape $j(j=1, m)$. This is obtained by picking up the corresponding columns in matrix $\mathcal{O}_{p+1}^{\prime}\left(\theta_{0}\right)$ in $(26)$, or equivalently in (51).

The counterpart of test (31), namely the directional or sensitivity test focussed on this mode $j$, writes:

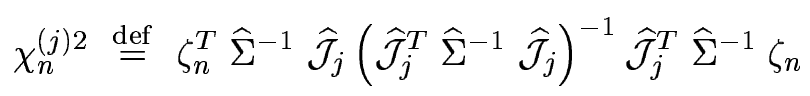

\section{Damage localization}

Damage localization consists in determining which part of the structure has been affected by the damage, more precisely which (groups of) elements of the structural parameters matrices (e.g. $M, C, K$ ) have changed. This problem is often addressed as an inverse estimation problem, based on an analytical model in the pre-damage stage and on modal identification in the post-damage stage. Typically, the deviations in the structural parameters corresponding to the observed deviation in the modal parameters are searched for using model updating techniques [27, 28, 29].

In the proposed approach, damage localization is stated as a detection problem instead. Of course, an analytical model is handled at this stage. The rationale is similar to the approach for the modal diagnosis problem above: having a (usually identified) reference modal parameter $\theta_{0}$ and a reference structural (FEM) analytical model in one hand, and a new data sample in the other, decide which structural parameters have deviated from their reference values.

\subsection{Residual sensitivities and residual uncertainty}

As for the damage detection and modal diagnosis problems, in a statistical framework the key issue is again to assess the significance of the residual sensitivity to specified structural changes w.r.t. the residual uncertainty.

As made explicit in (30), the mean value of residual $\zeta_{n}$ under the hypothesis $\mathbf{H}_{1}$ of a small deviation $\delta \theta$ in the system parameter $\theta$ from a reference value $\theta_{0}$, is:

$$
\mathbf{E}_{1}\left(\zeta_{n}\right)=\mathcal{J}\left(\theta_{0}\right) \delta \theta
$$

Under the assumption of small deviation again, the following relation holds:

$$
\delta \theta \approx \mathcal{J}_{\theta \Psi} \delta \Psi
$$

$\mathrm{RR} \mathrm{n}^{\circ} 4645$ 
where $\Psi$ is the vector of (FEM) structural parameters to be monitored, and $\mathcal{J}_{\theta \Psi}$ is the Jacobian matrix containing the sensitivities of the modes and mode-shapes w.r.t. those structural parameters.

In other words, the damage localization problem is addressed by plugging aggregated sensitivities of the modes and mode-shapes w.r.t. (FEM) structural parameters in the setting used for damage detection. Thus, plugging (35) into (34), we test, with the aid of a $\chi^{2}$-test again, whether the deviation in the residual $\zeta_{n}$ :

$$
\mathbf{E}_{1}\left(\zeta_{n}\right)=\mathcal{J}(\Psi) \delta \Psi
$$

where

$$
\mathcal{J}(\Psi) \stackrel{\text { def }}{=} \mathcal{J}\left(\theta_{0}\right) \mathcal{J}_{\theta \Psi}
$$

is significant or not. This results in directional tests, focussed in specific directions of the structural space, which perform the same type of damage-to-noise sensitivity analysis of the residual as for damage detection and modal diagnosis. These tests deliver damage diagnostics and localization information, without solving any inverse problem for model updating.

Of course, since the dimension of the structural parameter space is much higher than the dimension of the modal parameter space, the $\delta \theta$ in (35) are linearly dependent, even if the $\delta \psi$ 's are not. The idea $[30,31,6]$ is to cluster somehow the deviations $\delta \psi$ in the structural parameter space.

The steps of this damage localization approach are now described in detail. The off-line and on-board computation stages are distinguished, in 5.2 and 5.3 respectively.

\subsection{Off-line computations}

The off-line stage is devoted to the computation of the residual sensitivities w.r.t. structural changes. First we explain the different parameterizations and Jacobians which are needed for this purpose. Then we describe three design steps: computing the sensitivities (35), matching theoretical and actual sensitivities, aggregating the sensitivities.

\subsubsection{Computing the residual sensitivities to structural changes}

For computing the residual sensitivity w.r.t. structural changes given in (37), we need to compute the Jacobian $\mathcal{J}_{\theta \Psi}$ defined in (35).

For this purpose, in addition to the structural parameterization $\Psi$, two other

parameterizations are needed: $\Phi_{i}^{(d)} \stackrel{\text { def }}{=} \theta$, the set of the (discrete) identified modal 
parameters, and $\Phi_{a}$, the set of the (continuous) analytical modal parameters computed from (2). Since the former is a discrete time parameterization and the latter a continuous time parameterization, we also need the continuous time counterpart $\Phi_{i}$ of $\Phi_{i}^{(d)}$, obtained using (8). Moreover, the re-parameterization of the modes in terms of frequencies and damping coefficients are needed for both $\Phi_{i}$ and $\Phi_{a}$, which we note $\vartheta_{a}$ and $\vartheta_{i}$, respectively.

It should be stressed that, when the system is assumed to be conservative, namely $C=0$, which is often the case in FEM models, $\Phi_{a}$ contains all the frequencies, but not the damping coefficients, of the structure, and all the mode-shapes, which are real and usually mass-normalized. Also, the FEM mode-shapes have as many components as the total number of available sensors. Whereas $\vartheta_{i}$ contains those of the frequencies and associated mode-shapes contained in the signature $\theta_{0}$ which turn out to match with modes in $\vartheta_{a}$. Also, the mode-shapes have as many components as the actual number of sensors used and are not mass-normalized.

Altogether, the sensitivity $\mathcal{J}(\Psi)$ defined in (37) writes:

$$
\mathcal{J}(\Psi)=\mathcal{J}\left(\theta_{0}\right) \mathcal{J}_{\Phi_{i}^{(d)} \Phi_{i}} \mathcal{I}_{\Phi_{i} \vartheta_{i}} \mathcal{J}_{\vartheta_{i} \vartheta_{a}} \mathcal{I}_{\vartheta_{a} \Phi_{a}} \mathcal{J}_{\Phi_{a} \Psi}
$$

where:

- $\mathcal{J}_{\Phi_{i}^{(d)} \Phi_{i}}$ is the Jacobian of the transformation (8) of the discrete modes into the continuous ones;

- $\mathcal{I}_{\Phi \vartheta}$ is the Jacobian of the conversion (3) of the complex modes into continuous frequencies and damping coefficients, and $\mathcal{I}_{\vartheta \Phi}$ is the Jacobian of the inverse conversion;

- $\mathcal{J}_{\vartheta_{i} \vartheta_{a}}$ corresponds to the manual matching between the identified modes and the analytical (computed) ones;

- $\mathcal{J}_{\Phi_{a} \Psi}$ is the sensitivities of analytical modes to changes in structural parameters.

The Jacobians $\mathcal{J}_{\Phi_{i}^{(d)} \Phi_{i}}, \mathcal{I}_{\Phi \vartheta}$ and $\mathcal{I}_{\vartheta \Phi}$ are computed in the Appendix, whereas the derivation of $\mathcal{J}_{\Phi_{a} \Psi}$ and $\mathcal{J}_{\vartheta_{i} \vartheta_{a}}$ is addressed below in this section.

At this point, one comment is in order about the damage detection $\chi^{2}$-test in (31) and the modal diagnosis test in (33). Actually, we compute these tests using $\widehat{\mathcal{J}} \mathcal{J}_{\Phi_{i}^{(d)} \Phi_{i}} \mathcal{I}_{\Phi_{i} \vartheta_{i}}$ instead of $\widehat{\mathcal{J}}$. But this does not make any difference, since the two matrices $\mathcal{J}_{\Phi_{i}^{(d)} \Phi_{i}}$ and $\mathcal{I}_{\Phi_{i} \vartheta_{i}}$ are square invertible and cancel out in (31) and in (33).

$\mathrm{RR} \mathrm{n}^{\circ} 4645$ 


\subsubsection{Computing the sensitivities of analytical modes to structural changes}

The derivation of $\mathcal{J}_{\Phi_{a} \Psi}$ is described now. Differentiating the second equation in (2), we get $[30,31,32,33]$ :

$$
\delta \mu(2 \mu M+C) \phi+\left(\mu^{2} M+\mu C+K\right) \delta \phi+\mu^{2} \delta M+\mu \delta C+\delta K=0
$$

When the matrices $M, C, K$ are symmetric, pre-multiplying (39) with $\phi^{T}$, and using $\phi^{T}\left(\mu^{2} M+\mu C+K\right)=0$ which results from that symmetry, yield:

$$
\delta \mu=-\frac{\phi^{T}\left(\mu^{2} \delta M+\mu \delta C+\delta K\right) \phi}{\phi^{T}(2 \mu M+C) \phi}
$$

In case of an asymmetric system, the results of [34] should be used instead. They generalize the approach of [35], which is based on a complete modal basis. An overview on different types of approximation methods for handling incomplete modal bases can be found in [36].

Plugging (40) into (39) yields the following linear system in $\delta \phi$ :

$$
\left(\mu^{2} M+\mu C+K\right) \delta \phi=-\delta \mu(2 \mu M+C) \phi-\left(\mu^{2} \delta M+\mu \delta C+\delta K\right) \phi
$$

This system has no unique solution: if $\delta \phi$ is a solution, then $\delta \phi+\alpha \phi$, where $\alpha$ is a real constant, is also a solution. The solution $\delta \phi$ that is orthogonal to $\phi$ is selected, namely:

$$
\phi^{T} \delta \phi=0
$$

and then pre-multiplied by the observation matrix

$$
L \delta \phi
$$

since we are interested in the mode-shapes.

For each $\delta M, \delta C$ and $\delta K$, the equations (40), (41), (42) and (43) yield the corresponding change in the whole modal parameters set $\Phi_{a}$. This leads to the first order sensitivity relationship:

$$
\delta \Phi_{a}=\mathcal{J}_{\Phi_{a} \Psi} \delta \Psi
$$

where each column of the sensitivity matrix $\mathcal{J}_{\Phi_{a} \Psi}$ corresponds to a change in a structural parameter.

At this point, a comment is in order, on mode-shape normalization. As obvious from (41), the actual value of $\delta \phi$ depends on the normalization chosen for $\phi$. Therefore, it is important here to work with analytical mode-shapes normalized in the same way as the identified ones. Typically, the first component is fixed equal to one. 


\subsubsection{Matching theoretical and actual sensitivities}

How to match the changes in $\vartheta_{a}$ with the changes in $\vartheta_{i}$ is explained now.

It is well known that there is usually a discrepancy between the numerical modes $\vartheta_{a}$ provided by the analytical (FEM) model, and the identified modes $\vartheta_{i}$. Moreover, whatever the modal identification method is, there is an estimation error on $\vartheta_{i}$. Consequently, $\vartheta_{i} \neq \vartheta_{a}$ in general. However, this discrepancy between $\vartheta_{i}$ and $\vartheta_{a}$ is not crucial within our damage localization approach. Actually, $\vartheta_{i}$ is the reference modal signature and must be accurately determined, which is ensured by the subspace-based identification method, whereas $\vartheta_{a}$ is only used for computing a Jacobian matrix of sensitivities (change directions). A small error in such a direction enters the algorithm at a second order level in the residual $\zeta_{n}$. It is expected that this does not corrupt the localization delivered by the method.

Moreover, it should be noticed that the matching between $\vartheta_{i}$ and $\vartheta_{a}$ is generally partial. On one hand, only the first few natural modes are excited and/or observed, whereas a $p$ degrees of freedom finite element model yields $p$ modes, with $p$ often very large (several hundreds). Consequently, $\vartheta_{i}$ is at best strictly included in $\vartheta_{a}$. On the other hand, $\vartheta_{i}$ may contain modes which are not related to the eigen parameters of the structure. These modes appear in the presence of an harmonic excitation (as it is the case for rotating machineries for example [37]), or when the effect of the environment should be considered as an unknown colored noise, instead of a white noise as in (1).

Therefore, we assume that:

$$
\delta \vartheta_{i}=\mathcal{J}_{\vartheta_{i} \vartheta_{a}} \delta \vartheta_{a}
$$

where $\mathcal{J}_{\vartheta_{i} \vartheta_{a}}$ is a selection matrix which performs the correlation between the analytical (computed) modes and the identified ones.

\subsubsection{Aggregating the sensitivities}

The $(M, C, K)$ parameterization has generally many more parameters than the modal model. Thus, there are many more columns than lines in matrix $\mathcal{J}$ in (38). Moreover, using a small number of sensors, it is not reasonable to expect the discrimination

of all possible structural causes of a given deviation detected by the global damage detection test in (31).

To circumvent this difficulty, the idea is to aggregate the columns of $\mathcal{J}$ in (38) into clusters, which play the role of macro-failures, and for each cluster to define a barycentre, which plays the role of a Jacobian to be plugged in (31).

$\mathrm{RR} \mathrm{n}^{\circ} 4645$ 
The $\chi^{2}$-metric. In order to make the aggregation operation coherent with the $\chi^{2}$ decision stage, the metric chosen for performing the clustering is the metric of the $\chi^{2}$-test.

More precisely, let the $j$-th change direction be defined as the vector:

$$
\mathcal{J}_{j}=\widehat{\Sigma}_{n}^{-T / 2} \mathcal{J}\left(\theta_{0}\right) \mathcal{J}_{\Phi_{i}^{(d)} \Phi_{i}} \mathcal{I}_{\Phi_{i} \vartheta_{i}} \mathcal{J}_{\vartheta_{i} \vartheta_{a}} \mathcal{I}_{\vartheta_{a} \Phi_{a}} \mathcal{J}_{\Phi_{a} \Psi}(j)
$$

where $\mathcal{J}_{\Phi_{a} \Psi}(j)$ is the $j$-th column of $\mathcal{J}_{\Phi_{a} \Psi}$, and where $\widehat{\Sigma}_{n}^{-T / 2}$ is the 'square root' of the inverse of the covariance matrix $\widehat{\Sigma}_{n}$ :

$$
\widehat{\Sigma}_{n}^{-1}=\widehat{\Sigma}_{n}^{-1 / 2} \widehat{\Sigma}_{n}^{-T / 2}
$$

Note that such a decomposition is always possible since $\widehat{\Sigma}_{n}$ is guaranteed to be strictly positive definite from its numerical computation in (28).

Furthermore, we define the norm and the scalar product of the $\mathcal{J}_{j}$ 's as:

$$
\left\|\mathcal{J}_{j}\right\|^{2}=\mathcal{J}_{j}^{T} \mathcal{J}_{j}, \quad d_{i j}=\frac{\mathcal{J}_{i}^{T} \mathcal{J}_{j}}{\left\|\mathcal{J}_{i}\right\|\left\|\mathcal{J}_{j}\right\|}
$$

Removing the small vectors. Vectors with a low magnitude are likely to blur the results of the aggregation. Consequently, prior to clustering, small vectors are rejected using the following rule, based on the contrast (ratio) between the expectations of the directional test under no damage and small damage assumptions [6].

By definition, vector $\mathcal{J}_{j}$ corresponds to a change with rate 1 in physical parameter $j$. Therefore, up to a first order approximation, for a change with magnitude $\varrho$ in the $j$-th direction, the change vector is $\varrho \mathcal{J}_{j}$. The expectation of the corresponding sensitivity test (33) is equal to 1 under no damage hypothesis and to $\left(1+\varrho^{2} \mathcal{J}_{j}^{T} \mathcal{J}_{j}\right)$ under this damage hypothesis. The above mentioned contrast is thus:

$$
1+\varrho^{2} \mathcal{J}_{j}^{T} \mathcal{J}_{j}
$$

Consequently, considering that a damage with magnitude $\varrho$ in direction $\mathcal{J}_{j}$ is detectable provided that this contrast exceeds a threshold $\varepsilon_{1}$, the minimum magnitude of a damage for being detectable should be:

$$
\varrho_{\min }=\frac{\sqrt{\varepsilon_{1}-1}}{\left\|\mathcal{J}_{j}\right\|}
$$

A damage vector $\mathcal{J}_{j}$ will be rejected if this minimum damage magnitude cannot be reached, e.g. because greater than a percentage of variation on the physical parameters, namely if:

$$
\varrho_{\min } \geq \frac{\varepsilon_{2}}{100}
$$


Altogether, the rejection rule for small vectors writes:

$$
\left\|\mathcal{J}_{j}\right\| \leq 100 \frac{\sqrt{\varepsilon_{1}-1}}{\varepsilon_{2}}
$$

where $\varepsilon_{1}, \varepsilon_{2}$ are two thresholds selected by the user.

Clustering the remaining vectors. Since we are interested in change directions rather than change magnitudes, the change vectors to be clustered are normalized within this metric. Therefore, the aggregation process should work on the unit sphere, and a classification method able to handle this geometry is needed. For this reason, a vector quantization method $[38,39]$ of common use in speech processing has been chosen [6]. This method performs a hierarchical classification, while controlling the variability within the classes.

For each class, a barycentre $C_{j}$ is computed. This aggregation mechanism can thus be thought of as a statistical sub-structuring.

\subsection{On-board $\chi^{2}$-test for damage localization}

For performing damage localization, we should assess, for each barycentre $C_{j}$, whether the corresponding damage is significant or not. This problem is similar to the damage detection and modal diagnosis problems addressed in sections 3.4 and 4.2 respectively, and is solved in the same manner.

Because of (46), the following normalized residual should be considered:

$$
\widetilde{\zeta}_{n} \stackrel{\text { def }}{=} \widehat{\Sigma}_{n}^{-T / 2} \zeta_{n}
$$

Then the counterpart of the $\chi^{2}$-test in (33) is easily shown to write:

$$
\chi_{n}^{2}(j)=\widetilde{\zeta}_{n}^{T} \frac{C_{j} C_{j}^{T}}{\left\|C_{j}\right\|^{2}} \widetilde{\zeta}_{n}
$$

Its number of degrees of freedom is equal to $\operatorname{rank}\left(\mathcal{J}_{j}\right)$.

Assume that $\chi_{n}^{2}(j)$ exceeds a given threshold. Then, all the structural elements within the class corresponding to the barycentre $C_{j}$ are possible causes of the detected damage.

$\mathrm{RR} \mathrm{n}^{\circ} 4645$ 


\section{Discussion and relation to other works}

Several issues are addressed in this section, concerning the design and the handling of residuals for damage detection and localization.

First some further comments on the design of residuals for fault or damage detection are provided. Then the subspace-based damage detection residual introduced in section 3 is related to some other residuals, used for damage localization or model updating.

Residuals for fault detection. Model-based approaches to fault detection and isolation have been investigated, in $[40,41,42]$ to mention but a few. They build on discrepancies between process model outputs and measured outputs, generically called residuals, often generated as an output prediction error:

$$
\epsilon_{k}(\theta) \stackrel{\text { def }}{=} Y_{k}-\widehat{Y}_{k \mid k-1}(\theta)
$$

where $\widehat{Y}_{k \mid k-1}(\theta)$ is a one-step ahead prediction of the output data, computed on the basis of the parameterized model. The residual $\epsilon_{k}$ is then evaluated through a comparison with given thresholds. From a conceptual point of view, however, this type of residual suffers from the following limitation.

If the system is linear, written in an input-output or state-space form, and whatever the estimation method is, the prediction $\widehat{Y}$ is a linear combination of measured inputs and outputs. Stated otherwise, residual (48) is a first-order statistics in that case. But, from statistical inference theory, it is known that for performing inference about second-order characteristics - here, (modal) vibrating characteristics, or equivalently the eigenstructure of a linear state space system -, it is mandatory to use (at least) second-order statistics, that is covariances. Using linear combinations of the output data is not sufficient (in the statistical sense). This might be contrasted with some of the symptoms discussed in [43].

It should be noted that the subspace-based residual defined in (19) is actually a linear combination of the output covariances, and indeed is aimed at monitoring the system eigenstructure, as desired.

The above remark does not mean, however, that the prediction error (48) should not play any role in residual generation. As clearly stated in the system identification literature $[44,45,46]$, a parameter estimate should be updated with the aid of the gradient of the squared prediction error w.r.t. the parameter: $-1 / 2 \partial\left(\epsilon_{k}^{T}(\theta) \epsilon_{k}(\theta)\right) / \partial \theta$. And if the faults or damages affect the dynamics of the system, a residual built on that gradient is relevant too. 
Subspace-based residuals and other health monitoring residuals. We now comment on how the proposed subspace-based residuals relate to some other damage localization and model updating residuals in the literature.

In [1], damage detection is based on changes in the flexibility matrix computed as $\mathcal{F}=\Phi \Omega^{-1} \Phi^{T}$, where diagonal matrix $\Omega$, called modal stiffness matrix in the case of proportional damping, is the stiffness matrix of the single degree of freedom system resulting from decoupling of the equations by transformation to modal coordinates.

The damage locating vectors introduced in [5] are the last right singular vectors, namely the singular vectors associated with singular value 0 , of the change $\delta \mathcal{F} \stackrel{\text { def }}{=}$ $\mathcal{F}_{i}^{1}-\mathcal{F}_{i}^{0}$ in the measured flexibility matrix computed as $\mathcal{F} \stackrel{\text { def }}{=} K^{-1}$. In other words, these vectors form the kernel space of matrix $\Delta \mathcal{F}$. When viewed as loads on the system, they lead to stress fields identically zero over the damaged elements. This property is considered in [5] as a damage localization ability. An important limitation is that this is basically an input-output approach. However, the method can be extended to flexibility proportional matrices which can be computed from outputonly data [47].

On the other hand, the subspace-based residuals $\zeta$ defined in (19) are derived from the first left singular vectors of the observability matrix in modal basis $\mathcal{O}\left(\theta_{0}\right)$, from which matrix $S\left(\theta_{0}\right)$ in (19) is computed. In other words, for computing $\zeta$, we need the kernel space of matrix $\mathcal{O}^{T}\left(\theta_{0}\right)$.

One class of residuals used for model updating reviewed in [27] has the following form

$$
W\left(\theta_{i}-\theta_{a}-\mathcal{J}_{\Phi_{a} \Psi} \delta \Psi\right)
$$

where $W$ is a weighting matrix (design parameters), and subscripts $i, a$ have the same meaning as in 5.2.1. In other words, these residuals are based on discrepancies between identified and analytical modal parameters. This has to be contrasted with the residuals in (19) which handle altogether the identified modal parameters in $\theta_{0}$ and newly collected data in $\widehat{\mathcal{H}}$. These residuals do not involve any analytical model, and do not require any re-identification of the modal parameters on the new data. Moreover the $\chi^{2}$-test in (31) involves the precision in the estimated modal parameters [23]. This test statistics allows to assess whether a deviation in the reference modal parameter is significant w.r.t. the inherent inaccuracy in the modal parameter estimate.

In [28], strong emphasis is put on the importance of the physical meaning of the chosen parameterization. It has been argued above, however, that balancing this 
property with requirements on invariance w.r.t. changes in the state-space basis on the one hand, and on modal identifiability and detectability properties on the other one, provides us with other useful points of view.

Another overview of residuals used within model updating methods can be found in [48].

On the use and physical interpretation of the SVD. It is well known that one major byproduct of the singular value decomposition (SVD) is to provide us with various subspaces of interest, among which the right and left kernels (null space) of the considered matrix [18]. As highlighted in [43], the use of the SVD for the purpose of damage detection and localization is thus widespread. Moreover, the physical interpretation of the corresponding vectors is an important issue.

As mentioned above, the SVD is used in [5] for extracting the null space of the change $\delta \mathcal{F}$ in the flexibility matrix measured in both undamaged and damaged situations. The singular vectors are interpreted in terms of loads with null stress, and each singular value is interpreted as the difference in the external work done by the associated singular vector.

In the approach of this paper, the SVD is also used for extracting the null space of a matrix, namely the transpose of the observability matrix. Moreover, as explained in 3.1.3 and confirmed by extensive numerical experiments, the SVD helps in handling and overcoming non-stationary ambient excitation.

On the mode-shapes normalization. The last comment on the design and computation of residuals for health monitoring concerns the mode-shape normalization [36]. As outlined in 3.3.2, the proposed damage detection $\chi^{2}$-test is invariant w.r.t. mode-shape normalization, which turns out to be a useful property in practice. This has to be contrasted with the approach in [5].

Structural aggregation. Now some comments are in order on the statistical clustering of the sensitivities of the modes and mode-shapes w.r.t. FEM parameters described in section 5 .

It should first be noted, from (46), that noise-normalized sensitivities are handled in the proposed approach. Consequently, the norm and scalar product defined in (47) introduce a noise-normalized metric for assessing the size of the modes and modeshapes sensitivities to changes in structural parameters. Here noise refers to both measurement noise and modal estimation errors, as pointed out in 3.3.3. This has to be contrasted with the MAC (modal appropriation criterion) value, which is, in 
some sense, an absolute value: the MAC value does not take into account neither any measurement noise, nor any uncertainties in the modes and mode-shapes estimates (measured structural behavior).

Second, we stress that the statistical procedure described in 5.2.4 performs the aggregation of physical parameters, and thus generates what can be considered, in some sense, as super-elements. This procedure might be called statistical substructuring ${ }^{4}$.

\section{$7 \quad$ Application example}

In this section, some numerical results obtained with the proposed damage detection and localization method on a laboratory testbed called steel-quake are provided. Results obtained with the damage detection method on other real or laboratory examples can be found in $[49,52]$, together with some implementation issues. Some results obtained with the damage localization method on a real example can be found in [52].

The steel-quake structure is used at the Joint Research Centre in Ispra (Italy) to test the performance of steel buildings during earthquakes. The structure is a two-storey frame, excited with the aid of an impact hammer. The locations selected for the four impact tests are shown in Fig. 1. For each impact location, eight to ten experiments are performed, yielding data samples from the structure under different non-stationary excitations. Fifteen accelerometers are used for recording the reaction of the structure to the hammer impacts. The sampling frequency is $128 \mathrm{~Hz}$, and for each channel 3200 data points are recorded. The sensor locations are shown in Fig. 2. Damage scenarios have been generated using artificial seismic loading, and cracks appeared at different locations, in the vicinity of sensors $1,4,8,10$ and 13.

Damage detection. The damage detection test has been applied, using sensors $2,8,10,13$. The reference parameter $\theta_{0}$ has been identified on the data of the experiment $Q 10$; the frequencies and damping coefficients are given in Table 1.

The theoretical value of the $\chi^{2}$-test (31) under no damage assumption is 80 . The test has been computed on the data of the four undamaged scenarios $Q 09$ to $Q 12$, and on the data of the four damaged scenarios $Q 39$ to $Q 42$. The test values are given in Table 2, showing a clear discrimination between the undamaged and damaged cases.

\footnotetext{
${ }^{4}$ Substructuring is a procedure that condenses a group of finite elements into one element represented as a matrix (super-element).
}

$\operatorname{RR} \mathrm{n}^{\circ} 4645$ 


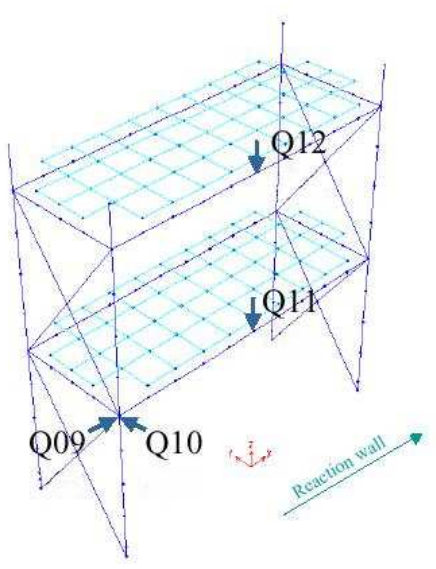

Figure 1: Impact locations Q09-12.

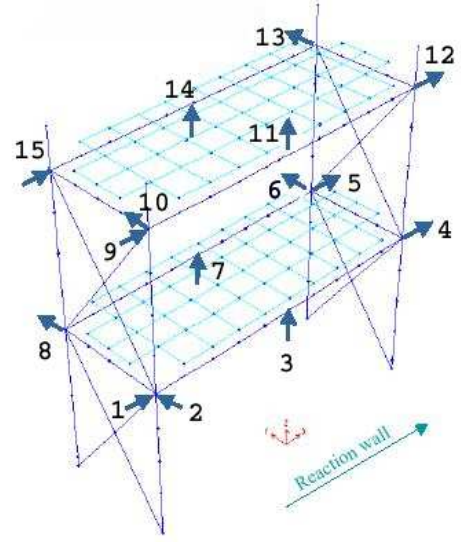

Figure 2: Sensors locations.

Table 1: Subspace identification - Some estimated modes (sensors 2/8/10/13, Q10).

\begin{tabular}{|l|c|c|c|c|c|c|c|c|}
\hline Mode & 1 & 2 & 3 & 4 & 5 & 6 & 7 & 8 \\
\hline Freq.(Hz) & 20.7728 & 14.4937 & 12.9889 & 11.8070 & 10.7943 & 9.6879 & 6.1302 & 3.9240 \\
Damp. & 0.2426 & 0.0195 & 0.0176 & 0.0610 & 0.2035 & 0.8905 & 0.0448 & 0.1803 \\
\hline
\end{tabular}

Table 2: Test values for undamaged and damaged scenarios.

\begin{tabular}{|c|c|c|}
\hline Scenario & Undamaged & Damaged \\
\hline Q09 /39 & $2.81 \cdot 10 \mathrm{e} 2$ & $3.78 \cdot 10 \mathrm{e} 6$ \\
\hline $\mathrm{Q} 10 / 40$ & $1.53 \cdot 10 \mathrm{e} 2$ & $2.20 \cdot 10 \mathrm{e} 7$ \\
\hline $\mathrm{Q} 11 / 41$ & $6.75 \cdot 10 \mathrm{e} 2$ & $2.18 \cdot 10 \mathrm{e} 4$ \\
\hline $\mathrm{Q} 12 / 42$ & $2.88 \cdot 10 \mathrm{e} 2$ & $1.62 \cdot 10 \mathrm{e} 4$ \\
\hline
\end{tabular}


Damage localization. The results reported here have been obtained using the new fully automated modal localization toolbox embedded in the Scilab Modal toolbox described in [53].

Damage localization has been performed using a rough FE model of the steelquake structure. This FE model has been obtained from the SAMCEF model used in COST F3, and updated using the Structural Dynamics Toolbox of E. Balmes. Although we advocate that using an inaccurate FE model is not critical for our localization method which handles modal changes directions, too large differences between the FE model and the structure may lead to localization errors. Moreover, the following comments are in order.

- Using that FE approximate model, we have been able to match the identified and computed mode-shapes for the first three modes only. Some work is still in progess for improving the FE model, in order to take into account mode-shapes of higher frequencies.

- The selected mode-shapes turn out to be in the X and Y directions only. This lack of information in the modal signature leads to localization inaccuracy in the $\mathrm{Z}$ direction.

- It also turns out, after careful comparison of the three mode-shapes in both the undamaged and damaged cases, that these mode-shapes have been hardly affected by the damage. Consequently the localization is to rely heavily on the frequencies, which is known not to be the most favorable situation for localization.

- Even though we use the first three correct mode-shapes only, for computing their sensitivities in (38), we must use the full mass and stiffness matrices, with all the inaccuracies due to the errors in the FE model.

Damage has been localized on the front, left and right piers around sensors 4,8 and 10. Some damages have been found around sensor 1 as well. In a preliminary experiment, damages have been found around the $Z$ sensors. But it turned out that the norm of the Jacobian columns associated with those events was very low, which could result in false alarms, and removing the $Z$ sensors removed the false alarms as well. The damage localization test did not perform well the discrimination between damaged elements situated on a same pier. It is expected that adding a mode-shape in the $Z$ direction will help in this respect. As a whole, the localization results obtained with this rough $\mathrm{FE}$ model have been satisfactory.

$\mathrm{RR} \mathrm{n}^{\circ} 4645$ 


\section{Conclusion}

We have addressed the damage localization problem stated as a detection problem and not as an inverse estimation problem. The proposed damage localization method is based on both a subspace residual and on a statistical analysis of aggregated sensitivities of the residual to the damages. We have described in detail the key components of the statistical damage detection and damage localization steps. We have distinguished the computations which can be performed off-line at a design stage and the computations which have to be done on-line while processing newly recorded data. We have related the proposed approach to several methods available in the literature. Numerical results obtained on a laboratory testbed (COST F3 steel-quake benchmark) have been provided.

\section{Acknowledgments}

The authors are indebted to Amine Hassim for providing the FEM and the structural matrices for the steel-quake example, to Yann Veillard for some computer implementations and experiments, and to Albert Benveniste for useful comments on an earlier version of the paper.

Part of this work has been carried out within the framework of the Eureka projects no 1562 Sinopsys (Model based Structural monitoring using in-operation system identification) coordinated by LMS, Leuven, Belgium, and no 2419 FliTE (Flight Test Easy), coordinated by Sopemea, Velizy-Villacoublay, France.

The covariance-driven subspace iodentification algorithm has been implemented within two toolboxes: the IN-OP module of the LMS software CADA-X, and the modal analysis module of the free INRIA software Scilab [51, 53]. The damage detection and localization methods have been implemented within the modal analysis module of Scilab, and are currently integrated within LMS software environment. 


\section{Appendix: Some Jacobian computations}

This appendix is twofold. First the sensitivity $\mathcal{J}\left(\theta_{0}\right)$ to modal changes given in $(25)$, in particular matrix $\mathcal{O}_{p+1}^{\prime}\left(\theta_{0}\right)$, is explicited in a manner which takes advantage of the real and imaginary parts of all complex numbers and vectors. Second, we compute the Jacobians $\mathcal{J}_{\Phi_{i}^{(d)} \Phi_{i}}$ of the transformation (8) of the discrete modes into the continuous ones, the Jacobian $\mathcal{I}_{\Phi \vartheta}$ of the conversion (3) of the complex modes into continuous frequencies and damping coefficients, and the Jacobian $\mathcal{I}_{\vartheta \Phi}$ of the inverse conversion.

\section{Computation of $\mathcal{O}_{p+1}^{\prime}\left(\theta_{0}\right)$ for $\mathcal{J}\left(\theta_{0}\right)$}

Since the modes and mode-shapes are pairwise complex conjugate, the observability matrix (15) writes

$$
\mathcal{O}_{p+1}(\theta)=\left(\begin{array}{ll}
\Phi & \bar{\Phi} \\
\Phi \Delta & \overline{\Phi \Delta} \\
\vdots & \vdots \\
\Phi \Delta^{p} & \overline{\Phi \Delta^{p}}
\end{array}\right)
$$

Taking advantage of the real and imaginary parts of the modes and mode-shapes, another matrix is introduced as follows:

$$
\widetilde{\mathcal{O}}_{p+1} \stackrel{\text { def }}{=}\left(\begin{array}{ll}
\Re(\Phi) & \Im(\Phi) \\
\Re(\Phi \Delta) & \Im(\Phi \Delta) \\
\vdots & \vdots \\
\Re\left(\Phi \Delta^{p}\right) & \Im\left(\Phi \Delta^{p}\right)
\end{array}\right)
$$

which results from a post-multiplication of $\mathcal{O}_{p+1}(\theta)$ by a permutation matrix.

Similarly, from $\theta$ defined in (10), define

$$
\widetilde{\theta} \stackrel{\text { def }}{=}\left(\begin{array}{l}
\Re(\Lambda) \\
\Re(\operatorname{vec} \Phi) \\
\Im(\Lambda) \\
\Im(\operatorname{vec} \Phi)
\end{array}\right)=\left(\begin{array}{l}
\Re(\theta) \\
\Im(\theta)
\end{array}\right)
$$

Now let

$$
\Theta_{p+1} \stackrel{\text { def }}{=} \operatorname{vec}\left(\begin{array}{l}
\Phi \\
\Phi \Delta \\
\vdots \\
\Phi \Delta^{p}
\end{array}\right)
$$

$\mathrm{RR} \mathrm{n}^{\circ} 4645$ 
Then

$$
\widetilde{\Theta}_{p+1} \stackrel{\text { def }}{=} \operatorname{vec} \widetilde{\mathcal{O}}_{p+1}=\left(\begin{array}{c}
\Re\left(\Theta_{p+1}\right) \\
\Im\left(\Theta_{p+1}\right)
\end{array}\right)
$$

Note that

$$
\widetilde{\mathcal{O}}_{p+1}^{\prime} \stackrel{\text { def }}{=} \partial \operatorname{vec} \widetilde{\mathcal{O}}_{p+1} / \partial \widetilde{\theta}=\partial \widetilde{\Theta}_{p+1} / \partial \widetilde{\theta}
$$

Using Cauchy-Riemann theorem, we get from (50) that

$$
\partial \widetilde{\Theta}_{p+1} / \partial \widetilde{\theta}=\left(\begin{array}{ll}
\partial \Re\left(\Theta_{p+1}\right) / \partial \Re(\theta) & \partial \Re\left(\Theta_{p+1}\right) / \partial \Im(\theta) \\
\partial \Im\left(\Theta_{p+1}\right) / \partial \Re(\theta) & \partial \Im\left(\Theta_{p+1}\right) / \partial \Im(\theta)
\end{array}\right)
$$

Therefore, for computing $\mathcal{O}_{p+1}^{\prime}\left(\theta_{0}\right)$, we proceed as follows:

- Compute $\mathcal{O}_{p+1}^{\prime}$ from (26) using only half of the modes and mode-shapes;

- Fill in $M \stackrel{\text { def }}{=}\left(\begin{array}{ll}\mathcal{O}_{p+1}^{\prime} & i \mathcal{O}_{p+1}^{\prime}\end{array}\right)$;

- Then

$$
\mathcal{O}_{p+1}^{\prime}\left(\theta_{0}\right)=\left(\begin{array}{c}
\Re(M) \\
\Im(M)
\end{array}\right)
$$

\section{Computation of $\mathcal{J}_{\Phi_{i}^{(d)} \Phi_{i}}, \mathcal{I}_{\Phi \vartheta}$ and $\mathcal{I}_{\vartheta \Phi}$}

Since none of the transformations (8) and (3) affect the mode-shapes, the restriction of the Jacobian to the eigenvectors is, in the three cases, the identity matrix with size $2 m r$. Thus, in each case, we first concentrate on the restriction of the Jacobians to the eigenvalues.

Computation of $\mathcal{J}_{\Phi_{i}^{(d)} \Phi_{i}}$. Using the notation:

$$
a=\Re(\mu), b=\Im(\mu), \quad x=\Re(\lambda), y=\Im(\lambda),
$$

the first equation of (8) writes:

$$
x=e^{\tau a} \cos (\tau b), \quad y=e^{\tau a} \sin (\tau b),
$$

from which we deduce:

$$
\left(\begin{array}{cc}
\frac{\partial x}{\partial a} & \frac{\partial x}{\partial b} \\
\frac{\partial y}{\partial a} & \frac{\partial y}{\partial b}
\end{array}\right)=\tau\left(\begin{array}{cc}
x & -y \\
y & x
\end{array}\right)=\tau\left(\begin{array}{cc}
\Re(\lambda) & \Re(i \lambda) \\
\Im(\lambda) & \Im(i \lambda)
\end{array}\right) \stackrel{\text { def }}{=} M_{\lambda}
$$


Then:

$$
\mathcal{J}_{\Phi_{i}^{(d)} \Phi_{i}}=\left(\begin{array}{cccc}
M_{\lambda_{1}} & & & \\
& \ddots & & 0 \\
& & M_{\lambda_{m}} & \\
0 & & & I_{2 m r}
\end{array}\right)
$$

Computation of $\mathcal{I}_{\Phi \vartheta}$. The relation (3) writes equivalently:

$$
a=\frac{-2 \pi f d}{\sqrt{1-d^{2}}}, \quad b=2 \pi f
$$

from which we deduce:

$$
\left(\begin{array}{cc}
\frac{\partial a}{\partial d} & \frac{\partial a}{\partial f} \\
\frac{\partial b}{\partial d} & \frac{\partial b}{\partial f}
\end{array}\right)=\left(\begin{array}{cc}
\frac{-2 \pi f}{\sqrt{\left(1-d^{2}\right)^{3}}} & \frac{-2 \pi d}{\sqrt{1-d^{2}}} \\
0 & 2 \pi
\end{array}\right) \stackrel{\text { def }}{=} A
$$

Then:

$$
\mathcal{J}_{\Phi \vartheta}=\left(\begin{array}{cccc}
A_{1} & & & \\
& \ddots & & 0 \\
& & A_{m} & \\
0 & & & I_{2 m r}
\end{array}\right)
$$

Computation of $\mathcal{I}_{\vartheta \Phi}$. The Jacobian of the relation (3) writes

$$
\left(\begin{array}{ll}
\frac{\partial d}{\partial a} & \frac{\partial d}{\partial b} \\
\frac{\partial f}{\partial a} & \frac{\partial f}{\partial b}
\end{array}\right)=\left(\begin{array}{cc}
\frac{-b^{2}}{\sqrt{\left(a^{2}+b^{2}\right)^{3}}} & \frac{-2 a b}{\sqrt{\left(a^{2}+b^{2}\right)^{3}}} \\
0 & \frac{1}{2 \pi}
\end{array}\right) \stackrel{\text { def }}{=} B
$$

Then:

$$
\mathcal{J}_{\vartheta \Phi}=\left(\begin{array}{cccc}
B_{1} & & & \\
& \ddots & & 0 \\
& & B_{m} & \\
0 & & & I_{2 m r}
\end{array}\right)
$$

$\mathrm{RR} \mathrm{n}^{\circ} 4645$ 


\section{References}

[1] A.K. Pandey and M. Biswas (1994). Damage detection in structures using changes in flexibility. Journal of Sound and Vibration, 169(1), 3-17.

[2] S.W. Doebling, C.R. Farrar, M.B. Prime and D.W. Shevitz (1996). Damage identification and health monitoring of structural and mechanical systems from changes in their vibration characteristics: a literature review. Los Alamos $N a$ tional Laboratory Report LA-13070-MS.

[3] H.G. Natke and C. Cempel (1997). Model-Aided Diagnosis of Mechanical Systems: Fundamentals, Detection, Localization, Assessment, Springer-Verlag.

[4] C.R. Farrar, S.W. Doebling and D.A. Nix (2001). Vibration-based structural damage identification. The Royal Society, Philosophical Transactions: Mathematical, Physical and Engineering Sciences, 359(1778), 131-150.

[5] D. Bernal (2002). Load vectors for damage localization. ASCE Journal of Engineering Mechanics, 128(1), 7-14.

[6] B. Gach-Devauchelle (1991). Diagnostic Mécanique des Fatigues sur les Structures Soumises à des Vibrations en Ambiance de Travail, Thesis, Paris IX Dauphine University (in French).

[7] R.P.C. Sampaio, N.M.M. Maia, J.M.M. Silva and A.M.R. Ribero (2000). On the use of transmissibility for damage detection and location. Proceedings of the European COST F3 Conference System Identification and Structural Health Monitoring, 363-376, Madrid, S.

[8] L. Hermans and H. Van der Auweraer (1999). Modal testing and analysis of structures under operational conditions: industrial applications. Mechanical Systems and Signal Processing, 13, 193-216.

[9] M. Basseville, A. Benveniste, M. Goursat, L. Hermans, L. Mevel and H. Van der Auweraer (2001). Output-only subspace-based structural identification: from theory to industrial testing practice. ASME Journal of Dynamic Systems, Measurement and Control, Special Issue on Identification of Mechanical Systems, $\mathbf{1 2 3}(4), 668-676$.

[10] D.J. Ewins (1984). Modal Testing: Theory and Practice, Research Studies Press, Letchworth, Hertfordshire, UK. 
[11] J.N. Juang (1994). Applied System Identification, Prentice Hall, Englewood Cliffs, NJ.

[12] L. Mevel, M. Basseville, A. Benveniste and M. Goursat (2002). Merging sensor data from multiple measurement setups for nonstationary subspace-based modal analysis. Journal of Sound and Vibration, 249(3), 719-741.

[13] D. Bernal (2000). Extracting flexibility matrices from state-space realizations. Proceedings of the European COST F3 Conference System Identification and Structural Health Monitoring, 127-136, Madrid, S.

[14] M. Basseville (1998). On-board component fault detection and isolation using the statistical local approach. Automatica, 34(11), 1391-1416.

[15] P. Stoïca and R.L. Moses (1997). Introduction to Spectral Analysis, Prentice Hall.

[16] H. Akaïke (1974). Stochastic theory of minimal realization. IEEE Transactions Automatic Control, AC-19, 667-674.

[17] A. Benveniste and J.-J. Fuchs (1985). Single sample modal identification of a nonstationary stochastic process. IEEE Transactions Automatic Control, AC30(1), 66-74.

[18] G. Golub and C.F. Van Loan (1996). Matrix Computations, Johns Hopkins University Press, Baltimore, MD (3rd ed).

[19] P. Van Overschee and B. De Moor (1996). Subspace Identification for Linear Systems: Theory - Implementation - Methods, Kluwer.

[20] M. Basseville, A. Benveniste, G.V. Moustakides and A. Rougée (1987). Detection and diagnosis of changes in the eigenstructure of nonstationary multivariable systems. Automatica, 23, 479-489.

[21] M. Viberg, B. Wahlberg and B. Ottersten (1997). Analysis of state space system identification methods based on instrumental variables and subspace fitting. Automatica, 33(9), 1603-1616.

[22] M. Basseville, M. Abdelghani and A. Benveniste (2000). Subspace-based fault detection algorithms for vibration monitoring. Automatica, 36(1), 101-109.

$\mathrm{RR} \mathrm{n}^{\circ} 4645$ 
[23] B. Delyon, A. Juditsky and A. Benveniste (1997). On the relationship between identification and local tests. IRISA Research Report 1104. ftp://ftp.irisa.fr/techreports/1997/PI-1104.ps.gz.

[24] Q. Zhang, M. Basseville and A. Benveniste (1994). Early warning of slight changes in systems and plants with application to condition based maintenance. Automatica, 30(1), 95-114.

[25] G.V. Moustakides and A. Benveniste (1986). Detecting changes in the AR parameters of a nonstationary ARMA process. Stochastics, 16, 137-155.

[26] M. Basseville (1999). An invariance property of some subspace-based detection algorithms. IEEE Transactions on Signal Processing, SP-47(12), 3398-3401.

[27] M. Link (1999). Updating of analytical models - Basic procedures and extensions. Modal Analysis and Testing, 281-304, J.M.M. Silva and N.M.M. Maia (Eds.), Kluwer Academic Publishers.

[28] M.I. Friswell, J.E. Mottershead and H. Ahmadian (2001). Finite element model updating using experimental test data: parameterization and regularization. Transactions of the Royal Society of London, Series A, Special Issue on Experimental Modal Analysis, 359, 169-186.

[29] C.P. Fritzen and K. Bohle (2001). Vibration-based global damage identification - A tool for rapid evaluation of structural safety. Proceedings of the 3rd International Workshop on Structural Health Monitoring, 849-859, Stanford, CA, Sept.12-14, 2001.

[30] G.V. Moustakides (1986). The problem of diagnosis with respect to physical parameters for changes in structures. IRISA Research Report 295.

[31] G.V. Moustakides, M. Basseville, A. Benveniste and G. Le Vey (1988). Diagnosing mechanical changes in vibrating systems. INRIA Research Report $\mathbf{9 4 2}$.

[32] U. Prells and M.I. Friswell (2000). Calculating derivatives of repeated and nonrepeated eigenvalues without explicit use of their eigenvectors. AIAA Journal, 38(8), 1426-1436.

[33] M.I. Friswell and S. Adhikari (2000). Derivatives of complex eigenvectors using Nelson's method. AIAA Journal, 38(12), 2355-2357. 
[34] S. Adhikari and M.I. Friswell (2001). Eigenderivative analysis of asymmetric non-conservative systems. Int. Jal for Numerical Methods in Engineering, 51, 709-733.

[35] R.L. Fox and M.P. Kapoor (1968). Rates of change of eigenvalues and eigenvectors. AIAA Journal, 6(12), 2426-2429.

[36] E. Balmes. Efficient sensitivity analysis based on finite element model reduction.

[37] M. Basseville, A. Benveniste, B. Gach-Devauchelle, M. Goursat, D. Bonnecase, P. Dorey, M. Prevosto and M. Olagnon (1993). Damage monitoring in vibration mechanics: issues in diagnostics and predictive maintenance. Mechanical Systems and Signal Processing, 7(5), 401-423.

[38] Y. Linde, A. Buzo and R.M. Gray (1980). An algorithm for vector quantizer design. IEEE Transactions on Communications, 38, 702-710.

[39] R.M. Gray (1984). Vector quantization. IEEE ASSP Magazine, 1, 4-29.

[40] R.J. Patton, P. Frank and R. Clark, eds. (1989). Fault Diagnosis in Dynamic Systems - Theory and Application, Prentice Hall Int., UK.

[41] J.J. Gertler (1998). Fault Detection and Diagnosis in Engineering Systems, Marcel Dekker.

[42] J. Chen and R.J. Patton (1999). Robust Model-based Fault Diagnosis for Dynamic Systems, Kluwer Academic Publishers.

[43] H.G. Natke and C. Cempel (2001). The symptom observation matrix for monitoring and diagnostics. Journal of Sound and Vibration, 248(4), 597-620.

[44] L. Ljung (1999). System Identification - Theory for the User, 2nd edition, Prentice Hall, NJ.

[45] T. Söderström (1994). Discrete-Time Stochastic Systems - Estimation and Control, Prentice-Hall Int., UK.

[46] A. Benveniste, M. Métivier and P. Priouret (1990). Adaptive Algorithms and Stochastic Approximations, Springer, New York.

[47] D. Bernal and B. Gunes (2002). Damage localization in output-only systems: a flexibility based approach. Proceedings of the 20th International Modal Analysis Conference.

$\mathrm{RR} \mathrm{n}^{\circ} 4645$ 
[48] H.G. Natke, G. Lallement, N. Cottin and U. Prells (1995). Properties of various residuals within updating of mathematical models. Inverse Problems in Engineering, 1, 329-348.

[49] L. Mevel, L. Hermans and H. Van der Auweraer (1999). Application of a subspace-based fault detection method to industrial structures. Mechanical Systems and Signal Processing, 13(6), 823-838.

[50] B. Peeters and G. de Roeck (2000). One year monitoring of the Z24-bridge: environmental effects versus damage events. Proceedings of the International Modal Analysis Conference, 1570-1576.

[51] M. Goursat, M. Basseville, A. Benveniste and L. Mevel (2000). A Scilab toolbox for output only modal analysis and diagnosis. Proceedings of the 18th International Modal Analysis Conference.

[52] L. Mevel, M. Goursat, M. Basseville (2003). Stochastic subspace-based structural identification and damage detection and localization - Application to the Z24 bridge benchmark. Mechanical Systems and Signal Processing, Special Issue on COST F3 benchmarks, 17, to appear.

[53] L. Mevel, M. Goursat, A. Benveniste (2003). Using subspace on flight data, a practical example. Proceedings of the 21th International Modal Analysis Conference, Kissimmee, Fl, Feb. 3-6, 2003. 


\section{Contents}

1 Introduction $\quad 3$

2 Modeling and parameterizations 4

2.1 Dynamical model and structural parameters . . . . . . . . . . 5

2.2 State-space model and canonical parameterization . . . . . . . . . 6

2.3 Different sensor types . . . . . . . . . . . . . . . . . 7

2.4 The damage detection and localization problems ....... 8

3 Damage detection 9

3.1 Residual associated with stochastic subspace identification . . . . . . 9

3.1.1 Output-only covariance-driven subspace identification . . . . 10

3.1.2 Exploiting a canonical parameter characterization . . . . . . . 11

3.1.3 On the effect of a truncated modal space . . . . . . . . . 13

3.2 Residual sensitivities and residual uncertainty . . . . . . . . . . . . . 14

3.3 Off-line computations . . . . . . . . . . . . . . . 14

3.3.1 Computing the residual sensitivities to modal changes . . . . 14

3.3.2 The sensitivity is invariant w.r.t. mode-shapes normalization 15

3.3.3 Computing the residual uncertainty . . . . . . . . . . 16

3.4 On-board residual analysis . . . . . . . . . . . . . . . . . . . . 16

3.4.1 The residual is Gaussian . . . . . . . . . . . . . 17

3.4.2 On-board $\chi^{2}$-test for damage detection . . . . . . . . . . 17

3.4.3 The $\chi^{2}$-test is invariant w.r.t. design matrices . . . . . . 17

4 Modal diagnosis $\quad 18$

4.1 Residual sensitivities and residual uncertainty . . . . . . . . . . . 18

4.2 On-board $\chi^{2}$-test for modal diagnosis . . . . . . . . . . . . 19

5 Damage localization $\quad \mathbf{1 9}$

5.1 Residual sensitivities and residual uncertainty . . . . . . . . . . . 19

5.2 Off-line computations . . . . . . . . . . . . . 20

5.2.1 Computing the residual sensitivities to structural changes . . 20

5.2.2 Computing the sensitivities of analytical modes to structural changes . . . . . . . . . . . . . . 22

5.2.3 Matching theoretical and actual sensitivities . . . . . . . . 23

5.2.4 Aggregating the sensitivities . . . . . . . . . . . 23

5.3 On-board $\chi^{2}$-test for damage localization . . . . . . . . . . 25 
6 Discussion and relation to other works

7 Application example

8 Conclusion

Acknowledgments

Appendix: Some Jacobian computations

References 


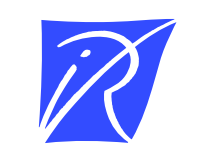

Unité de recherche INRIA Lorraine, Technopôle de Nancy-Brabois, Campus scientifique, 615 rue du Jardin Botanique, BP 101, 54600 VILLERS LÈS NANCY

Unité de recherche INRIA Rennes, Irisa, Campus universitaire de Beaulieu, 35042 RENNES Cedex

Unité de recherche INRIA Rhône-Alpes, 655, avenue de l'Europe, 38330 MONTBONNOT ST MARTIN

Unité de recherche INRIA Rocquencourt, Domaine de Voluceau, Rocquencourt, BP 105, 78153 LE CHESNAY Cedex

Unité de recherche INRIA Sophia-Antipolis, 2004 route des Lucioles, BP 93, 06902 SOPHIA-ANTIPOLIS Cedex

Éditeur

INRIA, Domaine de Voluceau, Rocquencourt, BP 105, 78153 LE CHESNAY Cedex (France)

http://www.inria.fr

ISSN 0249-6399 